\title{
DISTRIBUCIÓN DE SINSONTILLO (POLIOPTILA LEMBEYEI) \\ EN EL SUR DEL ORIENTE DE CUBA, IMPLICACIONES PARA SU CONSERVACIÓN
}

\author{
Distribution of Cuban Gnatcatcher (Polioptila lembeyei) \\ in the south-east of Cuba, implications for conservation \\ Carmen Plasencia-León ${ }^{1 \mathrm{a},}$, Nicasio Viña-Dávila², \\ Malbelys Padilla-Sánchez ${ }^{1 \mathrm{~b}}$ y Eduardo E. Iñigo-Elias ${ }^{3}$
}

\begin{abstract}
${ }^{1}$ Centro Oriental de Ecosistemas y Biodiversidad (BIOECO), José A. Saco No. 601, Santiago de Cuba, Cuba, C.P. 90100; ${ }^{\text {la }}$ (1) orcid.org/0000-0002-2868-8850; ${ }^{\text {b }}$ (D) orcid.org/0000-0002-6776-0843. ${ }^{2}$ Secretaría del Corredor Biológico en el Caribe, Santo Domingo, República Dominicana; (1) orcid.org/0000-0002-7353-6434. ${ }^{3}$ Cornell Lab 159 Sapsucker Woods Rd., Ithaca, NY 14850, USA. *Para correspondencia: p.lembeyei@gmail.com.
\end{abstract}

\section{RESUMEN}

El Sinsontillo (Polioptila lembeyei) es una especie endémica de Cuba que posee una distribución restringida y de la que se conoce poco de su historia natural. La presente investigación se desarrolló en el sur de Oriente Cubano donde la especie es relativamente abundante. En la actualidad se desconocen las características de su distribución, lo que dificulta implementar acciones de conservación efectivas. Se trabajó en una franja costera al sureste de Cuba de 377 km de largo entre Cabo Cruz y Punta de Maisí y 10 km de ancho máximo. Se muestrearon 141 localidades a partir del método de conteo por puntos con radio variable combinado con reclamos. Se obtuvo reportes de la especie en 85 localidades. Se comprobó la presencia de Sinsontillo cercano a los $400 \mathrm{~m}$ de altitud y a más de $8000 \mathrm{~m}$ de distancia de la línea costera. Se observó la especie en nueve formaciones vegetales, $88 \%$ de las localidades con presencia de la especie se agruparon en el matorral espinoso semidesértico costero, el bosque siempreverde micrófilo costero y prepostero (monte seco), la maleza compacta sin espinas y hierbas y pastos artificiales. Se realizó un modelo deductivo basado en las observaciones de campo, la literatura publicada y la experiencia de expertos. Las zonas de presencia ocupan $1063 \mathrm{~km}^{2}$ y está fragmentado en 274 parches de hábitat idóneo. Los valores de validación de modelo fueron buenos para el estadístico Kappa y para True Skill Statistic (TSS). El modelo predijo la presencia de la especie en una extensión inferior a la considerada en la bibliografía. Los resultados indican que los parches de hábitat idóneo de poca extensión presentes en zonas del sureste de Cuba son vulnerables a transformaciones por efectos de incidencia humana y del cambio climático y podría interrumpir la conectividad entre los parches de hábitats adecuados más grandes.

Palabras clave: Sinsontillo, Matorral Costero Espinoso, avifauna caribeña.

\section{ABSTRACT}

The Cuban Gnatcatcher (Polioptila lembeyei) is endemic to Cuba and has a restricted distribution. Little is known of this species beyond a few aspects of its natural history. This research was carried out in southeastern Cuba in areas where the species is relatively abundant. At present, the characteristics of its distribution are unknown, which makes it difficult to implement effective conservation actions. We studied gnatcatchers in the $377 \mathrm{~km}$ long $\mathrm{x}$ $10 \mathrm{~km}$ wide coastal strip between Cabo Cruz and Punta de Maisí, Cuba. We sampled a total of 141 localities using variable radius point counts combined with play-back, and obtained reports of the species at 85 localities. Cuban Gnatcatcher was found at an altitude nearly 
$400 \mathrm{~m}$ and at the distance of more than the $8000 \mathrm{~m}$ from the coastline. The specie was observed in nine plant cover, $88 \%$ of the locality's whit presence were grouped in coastal desert scrub, coastal and precoastal evergreen forest, compact wed without thorns and artificial grasses. A deductive model was made based on field observations, published literature and the expert's experience. The presence's area cover $1063 \mathrm{~km}^{2}$ and are fragmented in 274 patches of the suitable habitat. The validation values were good for the statistical test Kappa and True Skill Statistic (TSS). The model predicted the Cuban Gnatcatcher presence in smaller area than the considered in the literature. The results indicate that the small patches of suitable habitat present in the southeastern Cuba are vulnerable to transformation to the human effects and the climate change, which could interrupt the connectivity between the largest suitable habitat patches.

Keywords: Cuban Gnatcatcher, Cuban cactus scrub, Caribbean avian.

\section{INTRODUCCIÓN}

El género Polioptila (Familia: Polioptilidae, Aves), es exclusivo del continente americano y está representado con 12 especies, 11 de estas tienen áreas geográficas muy restringidas (Smith et al., 2018). La especie Polioptila caerulea es la única de amplia distribución, y tiene una distribución Neártica-Neotropical e inverna en el Caribe y América Central. Las especies presentes en Norteamérica ( $P$. californica, P. melanura, P. negricep y $P$. caerulea) han sido las más estudiadas (Atwood, 1980, 1988; Atwood y Bolsinger, 1992; Atwood et al., 1998; Atwood y Bontrager, 2001; Atwood y Lerman, 2006). Root y Yarrow (1967) realizaron trabajos de uso de hábitat para $P$. caerulea, durante la época reproductiva. Braden et al. (1997) investigaron las características de la vegetación dentro de los territorios de $P$. californica y su influencia en el éxito reproductivo. Recientemente Smith et al. (2018) analizó la distribución biogeográfica de la familia Polioptilidae a partir de la relación filogenética entre especies.

En Cuba están presentes las especies $P$. caerulea y $P$. lembeyei, esta última endémica. La distribución de $P$. lembeyei descrita por la literatura no es precisa. Garrido y Kirkconnell (2000), plantean que la especie $P$. lembeyei habita en vegetación costera. Atwood y Lerman (2006) la asocian a la ecorregión de Matorrales de Cactus de Cuba que presenta un área de unos $3300 \mathrm{~km}^{2}$. Los mapas de distribución en Garrido y Kirkconnell (2000) y Neotropical Birds Online (2010), presentan una extensión de presencia mayor, solo en el sur del oriente de Cuba supera los $4200 \mathrm{~km}^{2}$ y en la que se incluyen gran variedad de hábitats, no citados en Garrido y Kirkconnell (2000).

La carencia de estudios sobre esta especie cubana ha provocado contradicciones entre los autores al definir el área de distribución de $P$. lembeyei en Cuba. Las extensiones de presencia publicadas incluyen hábitats que la especie no utiliza. La falta de claridad en estos datos limitan la efectividad de acciones de conservación para la protección de la especie.

\section{OBJETIVO}

- Caracterizar la distribución del Sinsontillo (Polioptila lembeyei) en el sureste de Cuba e identificar aspectos a considerar en la planificación de la conservación de la especie.

\section{MATERIALES Y MÉTODOS}

El área de estudio se ubica entre Cabo Cruz y Punta de Maisí, con un ancho de diez kilómetros a partir de la línea de costa y una extensión de $4614 \mathrm{~km}^{2}$. El área seleccionada (Fig. 1) coincide con la extensión de ocurrencia de $P$. lembeyei en el sur de la región oriental reconocida por Garrido y Kirkconnell (2000) y de Neotropical bird (2010). 


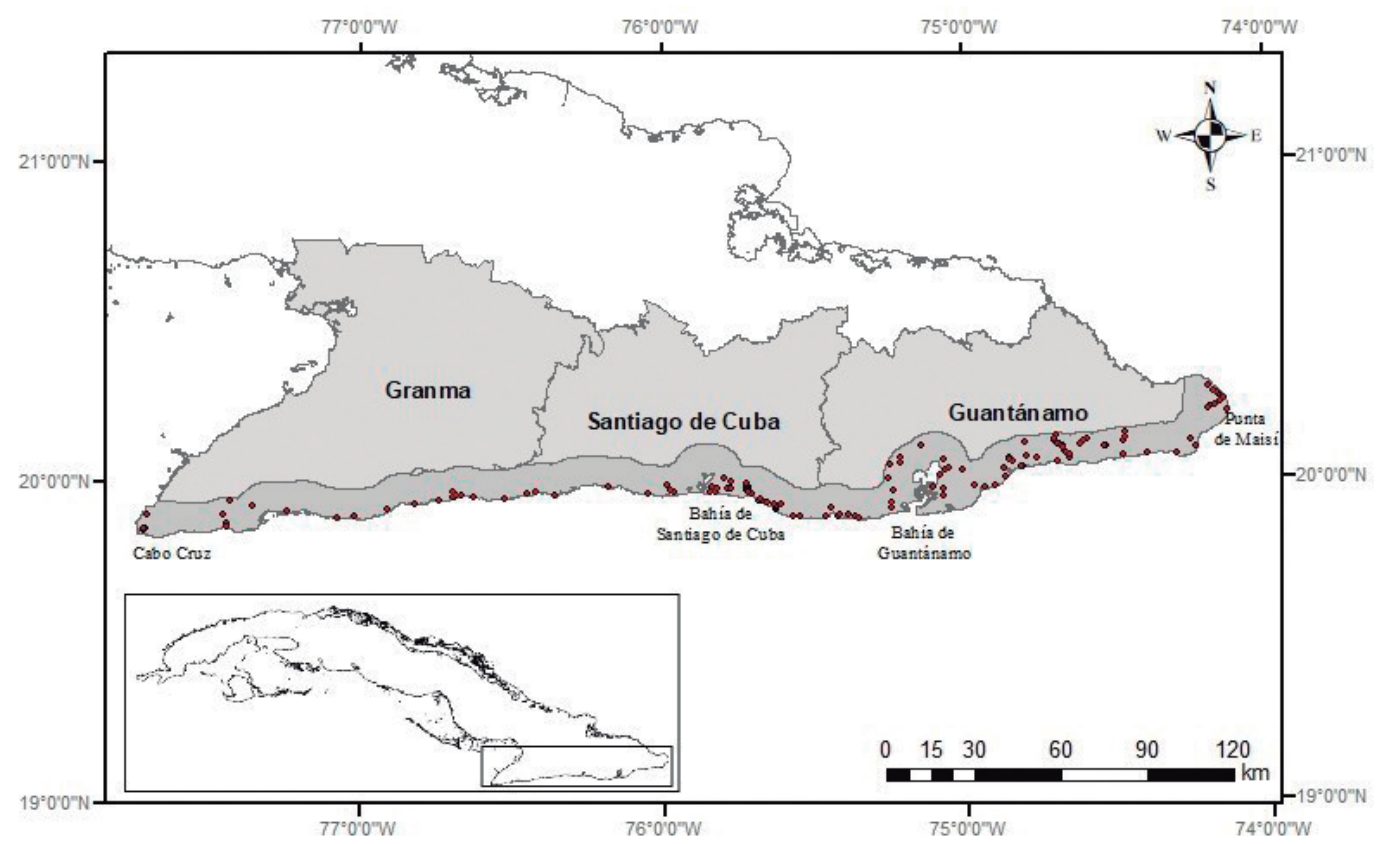

Leyenda

- Localidades estudiadas Provincias con zonas incluidas en el área de estudio Área de estudio Resto de Cuba

Figura 1. Ubicación de la zona de estudio.

Para conocer la distribución actual de $P$. lembeyei en el sur del oriente cubano se utilizó una combinación de métodos de muestreo. Se usó el método de conteo por puntos sin estimación de distancia propuesto por Wunderle (1994) y una vez concluido se realizaron reclamos acústicos (Oech y Oech, 1960; Johnson et al., 1981; Marion et al., 1981). El empleo de los dos métodos permite disminuir los falsos negativos pues la especie presenta alta frecuencia de respuesta a reclamos (Plasencia et al., 2019). Además, es posible extender el muestreo a todo el horario diurno dado que la especie responde de forma similar en todos los horarios del día y a cantos de otras localidades (Plasencia et al., 2019).

E1 muestreo se realizó durante el año 2014. Se trabajaron 141 puntos de muestreo, 81 de estos a lo largo de las carreteras y caminos cercanos a la línea costera y 60 restantes fueron en puntos ubicados perpendicular a la costa. La distancia mínima entre localidades fue de $143 \mathrm{~m}$ y la máxima de $13.5 \mathrm{~km}($ media $=2250 \mathrm{~m} ; \mathrm{DE}=2422 \mathrm{~m} ; \mathrm{N}=141)$. La distancia máxima (en línea recta) entre las localidades muestreadas es de $377 \mathrm{~km}$ entre Cabo Cruz y Punta Quemados en Maisí.

Todos los muestreos se realizaron entre las 6:30 y 17:00 horas, para muestrear la mayor cantidad de puntos en el menor tiempo posible. Cada punto de conteo fue georreferenciado y se tomaron datos de horario, fecha, altura y tipo de vegetación o cobertura (Wunderle, 1994).

Los primeros cinco minutos se realizaron detecciones visuales y acústicas de individuos siguiendo el método propuesto por Wunderle (1994) para determinar la presencia de aves en un hábitat. Si se observaba la especie antes de cumplirse el tiempo programado, se interrumpía el conteo y se tomaba el punto como positivo, en este caso no se aplicaba reclamo. Si finalizados los cinco minutos de observación no se detectaba ningún individuo de la especie se procedía al uso del reclamo. 
Se aplicaron dos reclamos de un minuto de duración y separados entre sí por dos minutos. En cada reclamo el equipo de reproducción se rotó en dirección de los puntos cardinales, prolongándose aproximadamente 15 segundos en cada dirección, de ser detectada la especie, se anotaba y se detenían las observaciones, si no se detectaba se detenía la observación trascurridos 5 minutos de culminado el segundo reclamo y se daba el punto como negativo. Para los reclamos se utilizó la grabación disponible en la Guía de cantos de aves de Cuba, elaborada por el Laboratorio de Ornitología de la Universidad de Cornell (CLO).

El volumen óptimo de reproducción se fijó a una intensidad que permitía ser escuchada por los investigadores a $50 \mathrm{~m}$ de distancia. Para esto se siguió el criterio sugerido por Atwood y Lerman (2006) que afirma que la vocalización del género Polioptila es perceptible al oído humano a esta distancia aproximada.

En el área de estudio se trabajaron 15 tipos de coberturas vegetales. Fueron muestreadas seis formaciones vegetales naturales cinco vegetaciones secundarias y cuatro cultivos, según el mapa de cobertura vegetal de Cuba, que utiliza la clasificación de vegetación de Capote y Berazaín (1984) modificada por Estrada et al. (2012).

Se compiló la información de presencia de $P$. lembeyei expuesta en las colecciones del Instituto de Ecología y Sistemática y del Museo Charles Randem de la Universidad de Oriente. Además se utilizaron los datos online de la base de datos eBird (eBird 2016).

Para determinar la distribución potencial de la especie se utilizó el método de modelación de clasificación de hábitats adecuados (C.H.A.). Se desarrolló un modelo deductivo de distribución basado en las observaciones de campo, la literatura publicada y la experiencia de expertos como sugiere Corsi et al. (2000). El procesamiento espacial se llevó a cabo con QGis 8.12.4.

Se utilizaron cuatro variables indirectas principales: Bases de cobertura vegetal de Capote y Berazaín (1984) con modificaciones de Estrada (2012), regiones naturales (Viña-Bayés, 2000), geología (formaciones geológicas), (Núñez, 2015) y cercanía a la línea de costa. De las bases utilizadas fueron eliminadas los asentamientos poblacionales y las carreteras del área. La validez del modelo obtenido como predictor de la presencia y ausencia de la especie se comprobó a partir de las pruebas Kappa (Cohen, 1960; Fielding y Bell, 1997) y TSS o "True Skill Statistic" (Allouche et al., 2006).

\section{RESULTADOS}

Hasta la realización de este estudio se había reportado la presencia de la especie en 36 localidades en Cuba, 18 se ubican en la costa sur de Oriente según la base de datos eBird (eBird, 2016), las colecciones del Instituto de Ecología y Sistemática, el Museo Charles Randem de la Universidad de Oriente y los reportes e informes publicados (Llanes et al., 1989-1999; Melián et al., 2005; González-Alonso et al., 2008; Suárez et al., 2008; Sánchez-Losada et al., 2015) El dato de presencia de la especie más antiguo encontrado fue de 1917. En el presente estudio se obtuvieron registros de detección de la especie en 85 localidades (60\% de las localidades muestreadas) de la costa sur Oriental, de ellos, 71 (83\% de las localidades positivas) son localidades nuevas.

Las localidades muestreadas tienen una distancia a la costa que varía entre $27 \mathrm{~m}$ y hasta los $8400 \mathrm{~m}$. El $50.5 \%$ de las localidades con presencia se encontraron a menos de $1000 \mathrm{~m}$ de la costa. Todas las localidades muestreadas ubicadas a menos de $100 \mathrm{~m}$ de distancia de la costa $(\mathrm{N}=11)$ tuvieron presencia de la especie. Las localidades con presencia a más de 
$1000 \mathrm{~m}$ de distancia de la costa, se distribuyeron en áreas cercanas de la Bahía de Santiago de Cuba $(\mathrm{N}=8)$ y entre la Bahía de Guantánamo y la Punta de Maisí, esta última con mayor importancia pues presenta el $80.9 \%$ de las localidades positivas ubicadas a más de $1000 \mathrm{~m}$ de distancia de la costa.

Las localidades con presencia de la especie se encontraron por debajo de $400 \mathrm{~m}$ de altitud. El $95.2 \%$ (81) se distribuyen entre 0 y $200 \mathrm{~m} \mathrm{~s}$. n. m., de estas, el $85.2 \%$ se localizan entre 0 y 100 m s. n. m. Entre los 201-400 m s. n. m. se encuentra el $5 \%$ (4) de los sitios con detecciones de $P$. lembeyei. Las cuatro localidades por encima de los $200 \mathrm{~m} \mathrm{~s}$. n. m. se encuentran en la zona de Yacabo Arriba, en la zona baja de la Sierra de Imías, en la provincia de Guantánamo.

La especie $P$. lembeyei no estuvo presente en todas las formaciones vegetales muestreadas $(\mathrm{N}=15)$ (Chi-Cuadrado $=93.3, \mathrm{p}<0.0001)$. Fue detectada en nueve tipos de cobertura (Tabla I), cuatro a formaciones vegetales naturales, cuatro vegetaciones secundarias y una cobertura cultural. E1 88\% de las localidades con presencia de la especie están agrupadas en cuatro tipos de cobertura vegetal, dos formaciones vegetales naturales: el matorral espinoso semidesértico costero, bosque siempreverde micrófilo costero y precostero (monte seco); una vegetación secundaria, la maleza compacta sin espinas, y en hierbas y pastos artificiales. El resto de las localidades con presencia (12\%), se encuentran en dos formaciones naturales: el bosque semideciduo micrófilo y el matorral costero con abundancia de suculentas; y tres vegetaciones secundarias: el bosque indiferenciados, maleza compacta con espinas y el matorral indiferenciado.

Tabla I. Localidades muestreadas y con presencia de Sinsontillo (P. lembeyei) en la costa sur oriental de Cuba por tipo de cobertura vegetal

\begin{tabular}{|l|c|c|c|}
\hline Tipo de cobertura & $\begin{array}{c}\text { Localidades } \\
\text { muestreadas }\end{array}$ & $\begin{array}{c}\text { Localidades con } \\
\text { presencia de } \\
\text { P. lembeyei }\end{array}$ & $\begin{array}{c}\text { \% de Localidades con presencia } \\
\text { de P. lembeyei }\end{array}$ \\
\hline Matorral semidesértico costero & 26 & 26 & 100 \\
\hline Maleza compacta sin espinas & 30 & 27 & 65.0 \\
\hline $\begin{array}{l}\text { Bosque siempreverde micrófilo } \\
\text { costero y precostero (Monte seco) }\end{array}$ & 20 & 13 & 33.3 \\
\hline Hierbas y pastos artificiales & 27 & 1 & 100 \\
\hline Bosque semideciduo micrófilo & 1 & 1 & 50.0 \\
\hline $\begin{array}{l}\text { Matorral costero con abundancia de } \\
\text { suculentas }\end{array}$ & 2 & 5 & 23.8 \\
\hline Bosque indiferenciados & 21 & 2 & 100 \\
\hline Maleza compacta con espinas & 2 & 1 & 50.0 \\
\hline Matorral indiferenciado & 2 & 1 & \\
\hline
\end{tabular}

El modelo de distribución potencial obtenido divide la zona de estudio en: zonas con presencia potencial de la especie y zonas sin presencia (Fig. 2). La zona con presencia potencial de $P$. lembeyei, ocupa una extensión de $1063 \mathrm{~km}^{2}$, que representa el $23 \%$ de la zona de estudio seleccionada y se está fragmentada en 274 parches de hábitat adecuado.

La validación del modelo de distribución obtenido es un paso importante para definir su utilización como una aproximación más exacta de la distribución de la especie. El resultado de la prueba "True Skill Statistic" (TSS) para el modelo arrojó valores de sensibilidad de 0.96 y de especificidad de 0.89 , estos indican que la distribución potencial de la especie propuesta 
responde adecuadamente tanto a las predicciones de presencia como de ausencia. El valor general del estadístico TSS tuvo un valor positivo de 0.85 que confirma lo anterior.

El estadístico Kappa (Kappa $=0.849 ; \mathrm{p}=0.046)$, aportó valores positivos y significativos para el modelo de distribución generado. La capacidad predictiva considerando los valores de kappa se considera "Muy buena", teniendo en cuenta que valores por encima de 0.8 han sido considerados por varios autores como un buen indicador del poder predictivo de los modelos (Pearce y Ferrier, 2000).

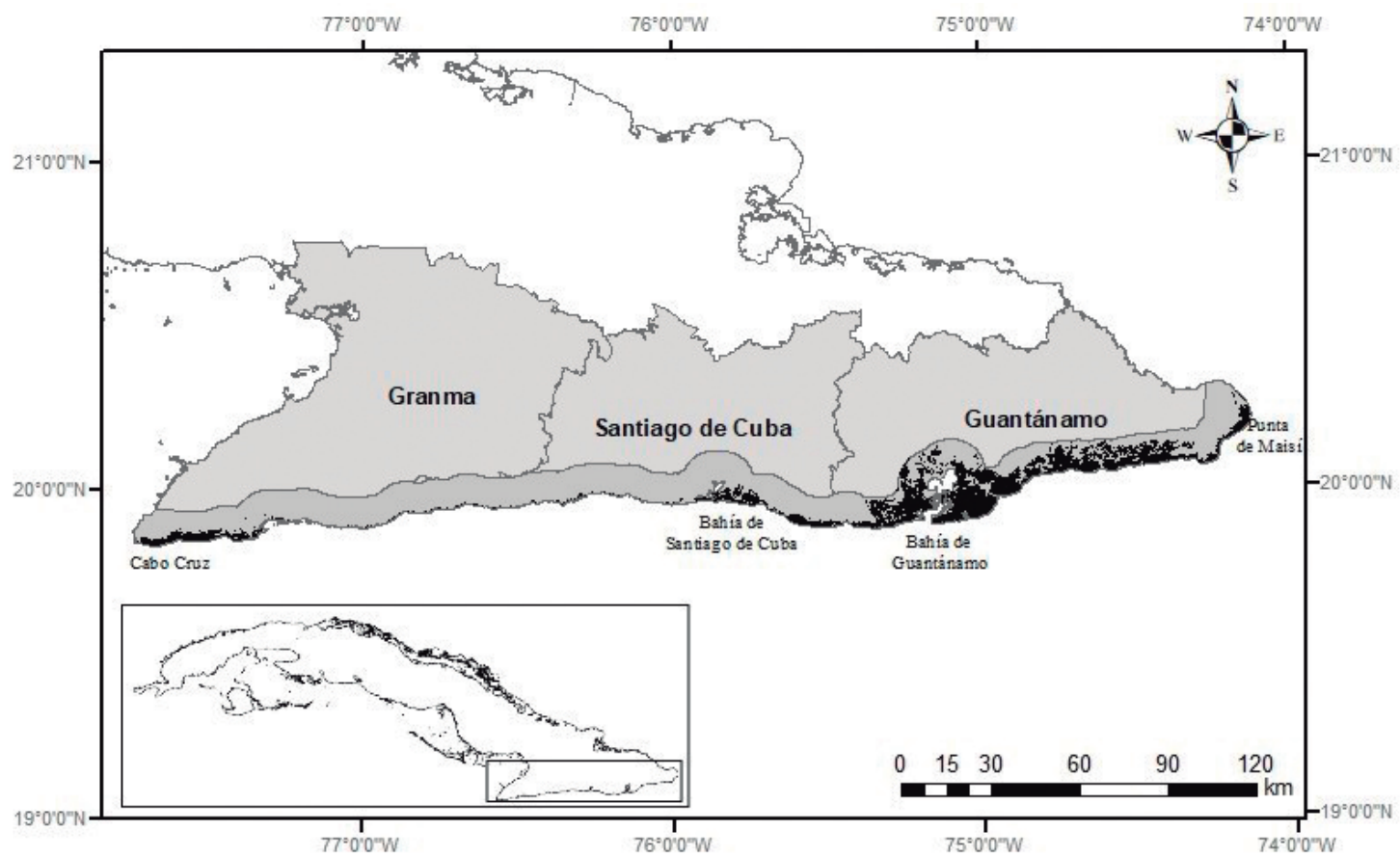

Leyenda

Áreas de distribución potencial de Sinsontillo Área de estudio

Provincias con zonas incluidas en el área de estudio Resto de Cuba

Figura 2. Modelo de distribución potencial de P. lembeyei en la costa sur oriental de Cuba indicando zonas de presencia potencial y zonas sin presencia de la especie.

Un análisis más detallado de la distribución potencial de $P$. lembeyei se obtiene considerando el tamaño, ubicación y distancia entre los parches de hábitats adecuados obtenidos. El parche de mayor extensión tiene $310.3 \mathrm{~km}^{2}$ y el menor $0.02 \mathrm{~km}^{2}$. Las áreas de hábitat adecuado de la especie con más de $1 \mathrm{~km}^{2}$ de extensión son $26(9.4 \%)$ y representan el $96.8 \%$ de la superficie de presencia del modelo, con un total de $1030.2 \mathrm{~km}^{2}$. Las zonas con hábitats adecuados de mayor importancia por su extensión son las terrazas costeras de Cabo Cruz, la Meseta de Santiago de Cuba y la zona entre Hatibonico y Maisí, que superan los $50 \mathrm{~km}^{2}$. Estas no son zonas compactas de hábitats homogéneos, aparece una fuerte imbricación con otros hábitats, que en su mayoría se debe a la transformación del hábitat por diversas actividades humanas como: ganadería ovino-caprina, pequeñas parcelas de cultivos anuales, caminos, casas aisladas y pequeños asentamientos. 
Los 248 parches de hábitat idóneo restantes presentan menos de un $\mathrm{km}^{2}$ de extensión, suman en total $33.2 \mathrm{~km}^{2}$ y representan el $3.2 \%$ de la extensión de distribución potencial total. Estas zonas están distribuidas a lo largo de toda el área de distribución potencial, pero son significativas en la zona entre Pilón y Santiago de Cuba donde no se presentan parches de extensiones superiores. Estos parches pequeños aparecen fundamentalmente en el sur de la Sierra del Turquino. Las características geográficas de la Sierra del Turquino indican que la distribución original debe haber estado restringida a una estrecha franja cercana a la costa y la posterior actividad humana como la construcción de caminos, actividades agrícolas y la urbanización, ha contribuido a la reducción de su hábitat optimo y a la fragmentación de los mismos.

La distancia entre parches de hábitat adecuado de la especie varía entre pocos metros y algo más de $2400 \mathrm{~m}$. La media de distancia entre zonas potenciales es $196 \mathrm{~m} \pm 279 \mathrm{~m}$ $(\mathrm{X} \pm \mathrm{DE}$; Máx $=2473$, Mín $=0, \mathrm{~N}=274)$. En la zona al sur de la Sierra del Turquino la

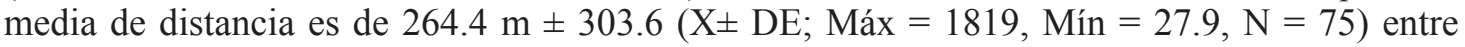
parches de hábitat adecuado. La combinación de tamaño pequeño, distribución lineal de las áreas y aislamiento de las áreas son factores que combinados incrementan la vulnerabilidad de las poblaciones de esta especie.

Una sola localidad con presencia de la especie no corresponde a el patrón de conducta general de preferencia de hábitat esperado. En la localidad de "La Aguadita", provincia Granma, se presenta una vegetación secundaria con elementos de bosque semideciduo micrófilo. En este caso no se tiene explicación para la presencia en este ecosistema y no se consideró en la definición del modelo.

\section{DISCUSIÓN}

La distribución de $P$. lembeyei en la costa sur de Oriente no se restringe a los hábitats presentes en el litoral, aunque la mayoría de las detecciones se encontraron a menos de $200 \mathrm{~m}$ de la costa. Estudios anteriores coinciden con que la especie utiliza los hábitats con presencia de vegetación xerofítica (Gundlach, 1876; AOU, 1998; Garrido y Kirkconnell, 2000; Atwood y Lerman, 2006). Existen áreas donde este tipo de vegetación se reduce a una franja muy estrecha paralela a la costa (Municipio de Guama, Santiago de Cuba), en otras se extienden hasta más de $8400 \mathrm{~km}$ de la costa (localidad de Yacabo Arriba, Guantánamo), (Viña-Bayés, 1998, 2000).

Polioptila lembeyei se distribuye regularmente entre $0 \mathrm{~m}$ y $200 \mathrm{~m}$ de altitud, en la zona de análisis. En cuatro localidades se detectó entre 200 y 400 m s.n.m. Estas se encuentran en la zona de Yacabo Arriba en la provincia de Guantánamo, ocupa parte del área denominada Sierra de Imías. En esta área por debajo de $500 \mathrm{~m}$ de altitud las condiciones climáticas se hacen extremas al disminuir las precipitaciones, lo que favorece el desarrollo de vegetación con características xerofíticas.

Atwood y Bolsinger (1992) determinaron la distribución altitudinal de Polioptila californica en Estados Unidos a partir de muestreos realizados en un periodo de 30 años. Solo el $3 \%$ de los sitios sobrepasa los $500 \mathrm{~m}$ de altitud. El $84 \%$ correspondió a localidades ubicadas por debajo de $\operatorname{los} 250 \mathrm{~m}$ de altitud y el $13 \%$ se localiza entre $\operatorname{los} 250 \mathrm{~m}$ y $500 \mathrm{~m}$ de altitud. Sus resultados sugieren que la distribución altitudinal de $P$. califórnica está limitada por la presencia del hábitat óptimo para sus poblaciones, porque, aunque el autor desconoce los factores ecológicos de selección, ha demostrado que su distribución esta obligatoriamente asociada al matorral xeromorfo (Atwood, 1980, 1988) y su distribución altitudinal está relacionada a la presencia de este hábitat. Se observó afinidad por el matorral más bajo (Atwood, 1980, 1988). 
Nuestros resultados coinciden con los obtenidos por Atwood y Bolsinger (1992). El principal patrón que determina la distribución altitudinal de $P$. lembeyei es la presencia de hábitats idóneos. Los resultados demuestran que la distribución altitudinal de esta especie está condicionada por la presencia de condiciones físico geográficas que permitan el desarrollo de vegetación xerofítica adecuada para la especie.

Las localidades con presencia de $P$. lembeyei se agruparon en formaciones vegetales con características xerofíticas que desarrollaron a partir de las características edáficas y climáticas de la zona de estudio. Se encontró en condiciones que van desde zonas descubiertas con arbustos aislados hasta zonas con árboles aislados y fuerte cobertura del estrato arbustivo. Resalta la presencia de la especie en nueve localidades identificadas como hierbas y pastos introducidos. En la zona de estudio los pastos no presentan las grandes extensiones típicas de los grandes llanos, son pequeñas zonas incluidas en una matriz de coberturas naturales y secundarias arbustivas. Un elemento significativo es que todos los casos positivos en esta cobertura estaban a menos de $100 \mathrm{~m}$ de áreas de condiciones muy propicias para la especie y siempre hubo presencia de arbustos.

En el modelo de distribución potencial de la especie, las áreas de ocupación más extensas de la especie en la costa sur oriental se encuentran en Cabo Cruz, provincia Granma y entre la localidad de Hatibonico y la Punta de Maisí, provincia Guantánamo. Estas zonas presentan extensas áreas, especialmente las que pertenecen a la provincia de Guantánamo, donde interactúan el conjunto de factores edafo-climáticos que propician el desarrollo de vegetación xerofítica. Actualmente no están representadas en su totalidad por matorrales naturales, existen parches de vegetación secundaria producto de la influencia humana, pero que mantienen las características xeromórficas. Los resultados alcanzados indican que el sur de la provincia de Guantánamo, mantiene una de las poblaciones más grandes de $P$. lembeyei. Las áreas de menor extensión en el modelo se encuentran comprendidas entre la localidad de Pilón, provincia Granma y la localidad de Morrillo Chico, en Santiago de Cuba.

La superficie de la distribución potencial generada por el modelo representa el $49.8 \%$ de la propuesta por Atwood y Lerman (2006). Estos autores afirman que la distribución de $P$. lembeyei en Cuba está asociada a la presencia de la ecorregión de matorrales de cactus cubano (World Wildlife Fund, 2009). La ecorregión tiene una extensión de $2132 \mathrm{~km}^{2}$ en el sur de Oriente según los mapas propuestos por World Wildlife Fund (2009). El área de coincidencia entre la ecorregión y la distribución potencial obtenida en nuestro estudio tiene una extensión de $852 \mathrm{~km}^{2}$ que representa el $80 \%$ de la superficie de distribución predicha en el modelo. El $20 \%$ de las áreas distribución potencial quedan por fuera de la ecorregión. Esto puede estar influenciado por la diferencia de precisión en las escalas que se utilizaron para elaborar los mapas de la ecorregión y los de la distribución potencial determinada el modelo.

La distribución potencial de $P$. lembeyei obtenida durante este estudio refleja el impacto de la actividad humana desarrollada en el área de estudio. Las características geográficas de áreas como las del sur de la Sierra del Turquino indican que la distribución original debe haber estado restringida a una estrecha franja cercana a la costa. Esta franja fue fragmentada y reducida por actividades como la construcción de viales, actividades agrícolas y urbanización.

La vulnerabilidad aumenta si consideran los escenarios de cambios climáticos previstos para la región, donde los periodos de sequías serán cada vez más extensos y la frecuencia se huracanes de gran intensidad se debe incrementar (Cambers, et al., 2008; Day, 2008; Suárez et al., 2008). Estos escenarios generan sobre áreas pequeñas varios impactos que afectan de manera directa a la especie, los incrementos de incendios naturales o provocados, 
la disminución de la disponibilidad de alimento y la disminución del éxito reproductivo. Estos efectos tendrán mayor impacto en poblaciones con hábitats muy pequeños y aislados como los analizados. Estos efectos provocan una fuerte presión en las poblaciones de $P$. lembeyei por disminución de hábitat y a la vez puede limitar la conectividad y generar aislamiento genético entre los parches de hábitat adecuado de mayor extensión. La distribución potencial tiene $464 \mathrm{~km}^{2}(43.7 \%)$ incluidos en alguna categoría de conservación y de ellos $358 \mathrm{~km}^{2}(77.2 \%)$, en las áreas más estrictas de conservación, quedan fuera de áreas protegidas $599 \mathrm{~km}^{2}$ $(56.3 \%)$. La distribución de zonas potenciales de presencia incluidas en áreas protegidas tiene dos elementos significativos, una mayor presencia de zonas incluidas en áreas protegidas en el tercio oriental de área de estudio (provincia de Guantánamo) y una muy baja representatividad entre Santiago de Cuba y Pilón.

La amenaza sobre las poblaciones de la especie en esta zona es aún más intensa si consideramos que en el sur de la Sierra del Turquino hay pocas zonas con presencia incluidas en las áreas protegidas actuales. Además, la especie no constituye un objeto de conservación, por no ser una especie amenazada y no haber sido considerados los elementos de conectividad con anterioridad.

En este caso es insuficiente establecer la conservación a partir de zonas protegidas, se requiere de otras herramientas de conservación como corredores, manejo de hábitat dentro y fuera de las áreas protegidas. El plan del SNAP (Centro Nacional de Áreas Protegidas, 2013) plantea tener el $10 \%$ de la distribución para el caso de las aves endémicas y amenazadas. Este criterio se cumple para la especie en la zona de Cabo Cruz y al este de la bahía de Santiago de Cuba en particular, pero no garantiza la conectividad de la especie y su intercambio genético entre las poblaciones de ambas zonas en el sureste de Cuba. La especie actúa en este caso como un indicador de lo que ocurre a nivel de la vegetación de matorral xeromorfo costero. Se hacen necesarias acciones de conservación fuera de áreas protegidas que garanticen la estabilidad de ecosistema y su conectividad, a partir de identificar objetos de conservación con una óptica más precisa.

\section{CONCLUSIONES}

El análisis de la distribución y características de las localidades con presencia de Polioptila lembeyei en el sur de oriente de Cuba demuestran que la especie ocupa fundamentalmente áreas cercanas a la costa pero que no se restringe a esta estando también presente en zona más alejadas y de mayor altitud donde las condiciones ecológicas propician la presencia de vegetación con características xeromórfica. El modelo de distribución obtenido predice que la especie Polioptila lembeyei presenta un área de distribución de menor extensión que la considerada en la literatura general.

Las áreas protegidas presentes en la costa sur oriental de Cuba actualmente apoyan la conservación de la especie pues incluyen áreas de ocupación importantes de las poblacionales de $P$. lembeyei, pero son insuficientes para garantizar la conectividad en entre ellos, lo que limita su efectividad en la conservación de la especie a largo plazo.

\section{AGRADECIMIENTOS}

Agradecemos al Centro Oriental de Ecosistemas y Biodiversidad, a la Universidad de La Habana por su apoyo con datos para este trabajo, a la Universidad de Oriente; al Instituto de Ecología y Sistemática y a IDEAWILD por su apoyo con equipamiento para la realización de la investigación. Al Laboratorio de Ornitología de Cornell por facilitar los cantos y su apoyo. 


\section{LITERATURA CITADA}

American Ornithologists' Union (A.O.U.). 1998. The A.O.U. check-list of the North American Birds. Washington, D.C., American Ornithologists' Union.

Allouche, O., A. Tsoar y R. Kadmon. 2006. Assessing the accuracy of species distribution models: prevalence, kappa and the true skill statistic (TSS). Journal of Applied Ecology, 43: $1223-1232$.

Atwood, J. L. 1980. The Unites States distribution of the California Black-Tailed Gnatcatcher. Western Birds, 11: 65-78.

Atwood, J. L. 1988. Speciation and geographic variation in black-tailed gnatcatchers. Ornithological Monographs, No. 42.

Atwood, J. L. y J. S. Bolsinger. 1992. Elevational distribution of California Gnatcatchers in the United States. Journal of Field Ornithology, 63: 159-168.

Atwood, J. L. y D. R Bontrager. 2001. California Gnatcatcher (Polioptila californica. The Birds of North America, No. 574.

Atwood, J. y S. B. Lerman. 2006. Family Polioptilidae (Gnatcatchers). In: Handbook of the birds of the world. Vol. 11. Barcelona: Lynx: 350-377 pp.

Atwood, J. L., S. H. TSai, C. A. Raynolds y M. R. Fugagli. 1998. Distribution and population size of California gnatcatcher on the Palos Verdes Peninsula, 1993-1997. Western bird, 29: $340-350$.

Braden, G., R. McKerman y S. Powell. 1997. Association of within-territory vegetation characteristics and the fitness components of California gnatcatcher. The Auk, 114 (4): 601-609.

Cambers, G., R. Claro, R. Juman y S. Scott. 2008. Climate change impacts on coastal marine of biodiversity in the insular Caribbean. Caribbean Natural Resources Institute, Technical report, No. 382 .

Capote, R. y R. Berazaín. 1984. Clasificación de las formaciones vegetales de Cuba. Revista Jardín botánico Nacional (La Habana), 5 (2): 27-75.

Centro Nacional de Áreas Protegidas. 2013. Plan del Sistema Nacional de Áreas Protegidas, 2014-2020. La Habana, Cuba, Ministerio de Ciencias Tecnología y Medio Ambiente: $161-163$.

Cohen, J. 1960. A coefficient of agreement for nominal scales. Educ. Psyschol. Meas, 20: 37-46.

Corsi, F., J. de Leeuw y A. Skidmore. 2000. Modelling species distribution with GIS. In: Research techniques in animal ecology: controversies and consequences. Columbia: University Press, New York, USA, 389-434 pp.

Day, O. 2008. The impacts of climate change on biodiversity in the Caribbean island: what we know, what we need to know, and building capacity for effective adaptation. Caribbean Natural Ressource Institute, Technical Report No. 386. 
eBird, 2016. eBird: a citizen-based bird observation network in the biological sciences [Online].

Disponible: http://www.ebird.org (accesado: 5 septiembre 2015).

Estrada, R., L. Martín, S. Rodríguez, I. Reyes, S. Galano, Y. Guerra, A. Batte, L. Mateo, C. Cabrera, R. Herrero, L. Castro, L. Coya, J. Almendares y L. Palenzuela. 2012. Mapa de cobertura forestal de la República de Cuba.

Fielding, A. y J. Bell. 1997. A review of methods for the assessment of prediction errors in conservation presence/absence models. Environmental Conservation, 24: 38-49.

Garrido, O. y A. Kirkconnell. 2000. Aves de Cuba. NY: Cornell Univ. Press, Ithaca, 200 pp.

González-Alonso, H., E. Perez-Mena, P. Rodriguez-Casariego, y O. Barrio-Valdez. 2008. Composición y abundancia de las comunidades de aves terrestres residentes y migratorias en Cayo Sabinal, Cuba. Poeyana, 496: 23-32.

Gundlach, J. 1876. Contribución a la ornitología cubana. La Antilla, La Habana, 364 pp.

Johnson, R., B. Brown, J. Simpson y L. Haight. 1981. Playback recordings as a special avian censuring technique. Studies in Avian Biology, 6: 68-75.

Llanes, A., A. González, B. Sánchez, D. Rodríguez, E. Pérez, P. Blanco, R. Oviedo y A. Pérez. 1989-1999. Estado de las comunidades de aves residentes y migratorias en ecosistemas cubanos en relación con el impacto provocado por los cambios globales. La Habana, Instituto de Ecología y Sistemática, 111 pp.

Marion, W. R., T. E. O’Meara y D. S. Maehr, 1981. Use Of Playback Recordings In Sampling Elusive Or Secretive Birds. Studies in Avian Biology, 6: 81-85.

Melián, L. O., D. F. Stotz, D. K. Moskovist y F. Rodríguez. 2005. Aves. In: Inventario Biológico Rápido Cuba: Siboney-Juticí. Santiago de Cuba: The Field Museun, 65-67 pp.

Neotropical Bird. 2010. Cuban Gnatcatcher (Poliopitla lembeyei). [Online] Disponible: http://neotropical.bird.cornell.edu/map/(accesado: 24 dieciembre 2010).

Núñez, K. 2015. Nuevo mapa geológico de Cuba a escala 1:1 000 000. La Habana, s.n.

Oech, V. y L. Oech. 1960. The use of recorded bird song as bird-censuring technique. Fliker, 32: 46-47.

Pearce, J. y S. Ferrier. 2000. Evaluating the predictive performance of habitat models developed using logistic regression. Ecological Modelling, 133: 225-245.

Plasencia, C., N. Viña y F. Tamayo. 2019. Evaluación del uso del reclamo pra identicar la presencia de Polioptila lembeyei (Aves: Polioptilidae). La Habana: Revista cubana de Ciencias Biológicas, 1-7.

Root, R. y R. Yarrow. 1967. A predator-decoy method for capturing insectivorous birds. The Auk 84, General Notes, 423-424 pp. 
Sánchez-Losada, M., A. E. Reyes-Vásquez, F. Rodríguez, G. López-Ibarra y N. Viña-Dávila. 2015. Biología reproductiva de Polioptila lembeyei (Aves: Polioptilidae) en la Reserva Ecológica Siboney-Juticí, Santiago de Cuba, Cuba. The Journal of Caribbean Ornithology, vol. 28: 6-10.

Smith, B. T., R. W. Bryson, W. M. Mauck, J. Chávez, M. B. Robins, A. Aleixo y J. Klicka. 2018. Species delimitation and biogeography of the gnatcatcher and gnatwrens (Aves: Polioptilidae). Molecular Phylogenetics and Evolution, 126: 45-57.

Suárez, A., D. Vilamajó, L. Mujica, J. Gerhartz, R. Capote y N. Blake. 2008. Climate change impacts on terrestrial biodiversity in the insular Caribbean. Caribbean Natural Reourses Institute Technical Report, 383 pp.

Viña-Bayés, N. 1998. Caracterización geográfica. In: Diversidad biológica del Macizo Montañoso Nipe-Sagua-Baracoa. Santiago de Cuba: Centro Oriental de Ecosistemas y Biodiversidad, 5-26.

Viña-Bayés, N. 2000. Caracterización geográfica de la Sierra Maestra. En: Diversidad Biológica del Macizo Montañoso Sierra Maestra. Santiago de Cuba, Cuba: Centro Oriental de Ecosistemas y Biodiversidad, 4-16.

World Wildlife Fund. 2009. Cuban cactus scrub. WildWorld Ecoregion Profile. National Geographic Society, p. 3.

Wunderle, J. 1994. Census Methods for Caribbean Land Bird. Unite State: Department of Agriculture, 3-7.

[Recibido: 10 de diciembre, 2019. Aceptado para publicación: 10 de marzo, 2020] 